\begin{tabular}{|c|l|}
\hline Title & Optimization of a magnetic refrigerator at room temperature for air cooling systems \\
\hline Author(s) & Kawanami, T suyoshi; Chiba, Kohtaro; Sakurai, Kohei; Ikegawa, Masahiro \\
\hline Citation & $\begin{array}{l}\text { International Journal of Refrigeration, 29(8), 1294 1301 } \\
\text { https://doi.org/10.1016/.ijrefrig.2006.07.018 }\end{array}$ \\
\hline Issue Date & $2006-12$ \\
\hline Doc URL & http://hdl.handle.net/2115/17234 \\
\hline Type & article (author version) \\
\hline File Information & IJR29-8.pdf \\
\hline
\end{tabular}

Instructions for use 


\title{
Optimization of a magnetic refrigerator at room temperature for air cooling systems
}

\author{
Tsuyoshi Kawanami, Kohtaro Chiba, Kohei Sakurai, Masahiro Ikegawa
}

\author{
Graduate School of Engineering, Hokkaido University \\ N13-W8, Kita-ku, Sapporo 060-8628, JAPAN \\ Corresponding author. Tel. : +81 117066427 \\ E-mail address : kawanami@eng.hokudai.ac.jp
}

\begin{abstract}
The purpose of this study is to understand the optimum operating condition of magnetic refrigerator at room temperature for direct air-cooling. The basic components of the target system are a magnetic circuit including two permanent magnets, a test section, an air blower, and an associated instrumentation. The test section consists of ten test cells which enclose gadolinium chips as a magnetic working substance in a prescribed packing rate. In order to change the applied magnetic field from 0 to $0.9 \mathrm{~T}$, the magnetic circuit is installed on an electric slider which generates reciprocating motion. The system performances are widely investigated both experimentally and analytically for the variety of conditions such as a volumetric flow rate of air, a packing length of magnetic working substance, and a heat exchange cycle. The results reveal that the present magnetic refrigerator has a maximum value of the cooling rate in an appropriate operating condition.
\end{abstract}

Keywords : Magnetocaloric effect, Magnetic refrigerator, Heat transfer, Particle bed

\section{Nomenclature}

$\begin{array}{lll}A & \text { cross sectional area of control volume } & \left(\mathrm{m}^{2}\right) \\ c_{p} & \text { specific heat } & \left(\mathrm{J} \mathrm{kg}^{-1} \mathrm{~K}^{-1}\right) \\ d_{p} & \text { particle diameter of magnetic material } & (\mathrm{m}) \\ F & \text { volumetric flow rate } & \left(\mathrm{m}^{3} \mathrm{~s}^{-1}\right) \\ h_{p} & \text { average heat transfer coefficient } & \left(\mathrm{W} \mathrm{m} \mathrm{K}^{-1}\right) \\ L_{m} & \text { packing length of working substance } & (\mathrm{m}) \\ M_{S} & \text { mass of magnetic working substance } & (\mathrm{kg}) \\ m & \text { mass flow rate } & \left(\mathrm{kg} \mathrm{s}^{-1}\right) \\ N & \text { number of collected data } \\ P r & \text { Prandtl number } & \\ Q & \text { amount of thermal energy } & (\mathrm{J}) \\ q & \text { cooling rate } & (\mathrm{W}) \\ T & \text { temperature } & \left({ }^{\circ} \mathrm{C}\right)\end{array}$




$\begin{array}{lll}t & \text { elapsed time } & (\mathrm{s}) \\ t_{e} & \text { heat exchange time } & (\mathrm{s}) \\ t_{m} & \text { change time of the magnetic field } & (\mathrm{s}) \\ U_{0} & \text { inlet velocity of air } & \left(\mathrm{m} \mathrm{s}^{-1}\right) \\ u & \text { gap velocity between particles } & \left(\mathrm{m} \mathrm{s}^{-1}\right) \\ V & \text { volume of control volume } & \left(\mathrm{m}^{3}\right) \\ x & \text { distance } & (\mathrm{m}) \\ \eta & \text { packing rate } & \\ \lambda & \text { thermal conductivity } & \left(\mathrm{W} \mathrm{m}^{-1} \mathrm{~K}^{-1}\right) \\ \mu & \text { dynamic viscosity } & \left(\mathrm{Pa} \mathrm{s}^{-1}\right) \\ \rho & \text { density } & \left(\mathrm{kg} \mathrm{m}^{-3}\right)\end{array}$

\section{Subscripts}

$\begin{array}{ll}\text { an } & \text { analytical } \\ \text { ave } & \text { average } \\ e x & \text { experimental } \\ f & \text { air } \\ i & \text { inlet, initial } \\ s & \text { magnetic working substance } \\ 0 & \text { initial }\end{array}$




\section{Introduction}

Magnetic refrigerators are capable of maintaining high efficiency at a range of extremely low temperatures at which operation is difficult for a conventional gas compression refrigerator. Such refrigeration devices are used to generate extremely low temperatures in the milli-Kelvin range. Accordingly because realization of a compact, low noise and low vibration refrigerator system is possible, it is expected that a magnetic refrigerator can be developed for a compact residential refrigeration system that can be operated in the room-temperature range.

However, at room temperature, the heat capacity of the magnetic working substances - the refrigerants - becomes markedly larger than at the extremely low temperatures at which they are typically used. The temperature distribution of the magnetic working substance obtained by changing the magnetic field becomes smaller. Therefore, efficient heat transfer control is important for constructing a useful magnetic refrigeration system. Improvement of the refrigeration cycle and system miniaturization have been studied [1-4] to solve this problem and to put room-temperature magnetic refrigerators to practical use, but no technology has been established. Urgent studies are necessary to obtain a practical system. The performance of the magnetic refrigeration system depends on the magnetocaloric effect of the magnetic working substance. Therefore, research and development of magnetic substances $[5,6]$ that can provide markedly higher magnetocaloric effects have been the subjects of great effort. Nevertheless, no examples exist of research and development from the perspective of promoting heat transfer of magnetic substances.

Accordingly, the primary objective of the present study is to elucidate heat transfer characteristics between magnetic working substances used for magnetic refrigeration systems and heat transfer media under a variety of conditions. In this study, a magnetic room-temperature refrigeration system is constructed, and an experimental study is executed to explain the influences of various factors on operating performance. In addition, a study of optimum operating conditions of the refrigeration system at room temperatures is carried out through numerical analysis.

\section{Experimental apparatus and procedure}

\subsection{Apparatus}

Figure 1 shows a schematic of the experimental apparatus used for this study. This experimental apparatus consists of a test section, a reciprocating drive system with permanent magnets, and an air transfer system. Figure 2 presents a detailed drawing of the test section used for this experiment.

The test section comprises 10 test cells (each $4 \mathrm{~mm}$ high, $8 \mathrm{~mm}$ wide, and 60 $\mathrm{mm}$ long) and gadolinium packing chips as a magnetic working substance. In addition, it has 12 dummy cells with 1-mm-diameter packed iron particles for maintaining the motion of the magnetic circuit smoothly, and an acrylic supporter for the test cells. A temperature measurement component is included to measure the air temperature at the test cell outlets. In this experiment, the third cell from the right end of the cell lines of the test section is assumed as a cell for temperature measurement and evaluation of characteristics. We arranged 0.1-mm-diameter T-type thermocouples to measure the temperature at the centers of the target cell outlets. A reciprocating drive system with permanent magnets consists of a magnetic circuit providing the magnetic field to the test cells. An electric slider allows the transfer of the magnetic circuit in a voluntary 
transfer distance, velocity, and timing. This magnetic circuit, which consists of two opposite permanent magnets arranged in a $10 \mathrm{~mm}$ interval and a yoke, is a closed circuit to prevent the magnetic field from leakage to the outside. The maximum concentration of the magnetic fluxes in a center between the permanent magnets is $0.9 \mathrm{~T}$.

\subsection{Procedure}

In the experiment, a blower first drives air. The flow rate of air charged into the test section is set at a prescribed value using a valve and a flow meter. The air temperature is set at a prescribed initial value using a heater and a cooler; it is confirmed that air temperature at both the inlet and the outlet of the measuring cell reached a steady state. After that, the electric slider, which is programmed with a personal computer for motion of the magnetic circuit in a prescribed transfer distance, transfer speed, transfer time, and stationary time, is started. The electric slider proceeds into a demagnetization process by motion in a prescribed transfer time (equivalent to the change time of the magnetic field $t_{m}$ ); subsequently, it stops for a heat exchange time $t_{e}$. It then proceeds to a magnetization process through a reverse motion. After that, the electric slider stops again for heat exchange time $t_{e}$; thereby, one cycle completes. The air temperature is recorded every $0.1 \mathrm{~s}$ for 10 cycles from the initiation of the electric slider motion.

In this present study, the packing length of the working substance $L_{m}$ is varied from $20 \times 10^{-3}$ to $60 \times 10^{-3} \mathrm{~m}$ based on the dimensions of the magnet (see Fig.2). The airflow rate $F$ per test cell is changed from $1.7 \times 10^{-5}$ to $20.0 \times 10^{-5} \mathrm{~m}^{3} \mathrm{~s}^{-1}$ due to the maximal power of the air blower. Furthermore, the initial air temperature $T_{0}$ and the transfer time of the magnetic circuit $t_{m}$ are set as $20^{\circ} \mathrm{C}$, and $0-30 \mathrm{~s}$, respectively. Besides, the packing length of the working substance $L_{m}$ is proportional to the mass of the magnetic working substance $M_{s} ; L_{m}=20 \times 10^{-3} \mathrm{~m}$ is equivalent to $M_{s}=1.5 \times 10^{-3} \mathrm{~kg}$.

\subsection{Data reduction}

In this experiment, thermal energy $Q_{e x}$ transferred to the air during the demagnetization process is calculated according to Eq.(1). The difference between outlet air temperature at that time $T_{f i}$ and the average value of air temperature change during in one cycle $T_{\text {fave }}$ was integrated from $i=1$ to $N$, and the mass flow rate of air $m_{f}$ the specific heat $c_{p f}$, and the period during the demagnetization process $\left(t_{m}+t_{e}\right)$ are multiplied.

$$
Q_{e x}=m_{f} c_{p f}\left(t_{m}+t_{e}\right) \sum_{i=1}^{N}\left(T_{f i}-T_{f a v e}\right)
$$

where $N$ denotes the total number of collected data during the demagnetization process. On the other hand, theoretical absorption thermal energy $Q_{a n}$ of the magnetic working substance based on the magnetic field change is calculated from the magnetic entropy change in the following equation:

$$
Q_{a n}=M_{s} T_{0} \Delta S_{m}(T, B),
$$

in which magnetic entropy change $\Delta S_{m}(T, B)$ per unit mass of the magnetic working substance is obtained by applying the external magnetic field. The magnetic entropy 
change is a function of absolute temperature $T$ and applied magnetic field $B$, as shown in the following equation.

$$
\Delta S_{m}(T, B)=\int_{0}^{B}\left[\frac{\partial M(T, B)}{\partial T}\right] d B
$$

Therein, $\partial M(T, B) / \partial T$ is given by molecular field approximation [7], in which the magnetic entropy in a simple crystal grid of the magnetic substance is calculated by an average value of the angular momentum inherent in all unpaired electrons. Cooling rate $q_{e x}$ and $q_{a n}$ in the experiment and the analysis were calculated by dividing heat exchange quantities $Q_{e x}$ and $Q_{a n}$ by time required for one cycle.

\section{Numerical simulation}

\subsection{Simulation model}

In addition to the experimental study, the optimum operating conditions of the system were examined by a numerical analysis. Basic assumptions for the used model are shown in Fig.3. The packing rate $\eta$ of the working substance was defined by assuming a gap $\delta$ between adjacent particles of the working substance.

$$
\eta=\frac{\pi}{6}\left(\frac{d_{p}}{d_{p}+\delta}\right)
$$

The distance to the flow direction of the control volume is given as particle diameter $d_{p}$. To represent the test cells, the flow path shape is assumed as a rectangular parallelepiped with inner dimensions of $4 \mathrm{~mm}$ height and $8 \mathrm{~mm}$ width. The particle diameter $d_{p}$ is set so that average mass of a foil-like magnetic working substance used for the experiment is equal to the mass of the working substance particles in the simulation.

\subsection{Governing equation}

The governing equation in this analysis is an energy conservation equation of the magnetic working substance (solid phase particles) and heat transfer media (gas phase). It is expressed as a function of the main direction $x$ as follows.

$$
\begin{aligned}
& \frac{\partial T_{s}}{\partial t}=\beta_{s}\left(T_{f}-T_{s}\right)+\frac{\dot{q}_{s}}{c_{p s}} \\
& \frac{\partial T_{f}}{\partial t}+u \frac{\partial T_{f}}{\partial x}=\alpha_{f} \frac{\partial^{2} T_{f}}{\partial x^{2}}+\beta_{f}\left(T_{s}-T_{f}\right)
\end{aligned}
$$

In the above equations, $\alpha_{f}$ is the thermal diffusivity of the gas phase and $\dot{q}_{s}$ is the rate of energy generation per unit volume of the medium. Furthermore, $\beta_{s}$ and $\beta_{f}$ respectively represent the factors expressed in the following: 


$$
\beta_{s}=\frac{A h_{p}}{\rho_{s} c_{p s} V_{s}}, \quad \beta_{f}=\frac{A h_{p}}{\rho_{f} c_{p f} V_{f}},
$$

where $\rho, A$, and $\mathrm{V}$ are a density, the cross sectional area of the control volume, and the volume of the control volume, respectively. In addition, $h_{p}$ is the average heat transfer coefficient between the fluid and particles. It is defined by the Ranz equation [8] expressing heat transfer in a particle layer in the following equation.

$$
h_{p}=\frac{\lambda_{f}}{d_{p}}\left[2.0+0.6 \operatorname{Pr}^{1 / 3}\left(\frac{d_{p} \rho_{f} u}{\mu_{f}}\right)^{1 / 2}\right]
$$

where $\lambda_{f}, \mu_{f}$, and Pr denote the thermal conductivity, the dynamic viscosity, and the Prandtl number of the fluid gas, respectively. Therein, $u$ is a gap velocity of fluid flowing between particles; it is given by the following equation.

$$
u=\frac{U_{0}}{1-\frac{\pi}{2 \sqrt{3}}\left(\frac{d_{p}}{d_{p}+\delta}\right)}
$$

\section{Results and discussion}

\subsection{Temperature change of a magnetic working substance}

Before some investigations concerning heat transfer characteristics was started, the research on adiabatic temperature change $\mathrm{D} T_{s}$, which occurred inside of the magnetic working substance when the magnetic field was removed from the magnetic working substance, has been done as shown in Fig.4. The horizontal axis shows the initial temperature $T_{0}$ of the magnetic working substance. Regarding the temperature change of the magnetic working substance, Fig. 4 shows that the initial temperature of the demagnetization process tends to show its maximum value at $20^{\circ} \mathrm{C}$, presumably because the Curie point of the magnetic working substance is $20^{\circ} \mathrm{C}$. Thereby, the magnetocaloric effect of the working substance and its cooling energy become maximal.

\subsection{Temperature distribution of air at one cycle}

To verify the validity of the analytical model, the experimental results and the analytical ones are compared in this section. Figure 5 shows experimental results of air temperature at the outlet of the measuring cell for the heat exchange time $t_{e}=30 \mathrm{~s}$. Analytical results in the same condition are shown in Fig.6. Elapsed time $t$ of the horizontal axis shows one cycle: the starting time of the sixth cycle is assumed as $0 \mathrm{~s}$. The figures illustrate the air temperature to decrease during demagnetization process and to increase during magnetization one in all conditions of airflow rates with both experimental and analytical results. Those results imply that the working substance absorbs heat from ambient air during the demagnetization process and discharges heat to the ambient air during the magnetization due to the magnetocaloric effect. Thereby, the higher the airflow rate, the larger the temperature change in the demagnetization process and immediately after magnetization. The gap velocity $u$ between adjacent particles is higher for higher airflow rates. For that reason, heat transfer between the 
working substance and air is promoted and heat exchange with air takes place in a shorter time. For the same reason, when an airflow rate becomes larger, the air temperature tends to approach the initial temperature in a short heat exchange time. From these results, the analytical model constructed in this study well expresses the experimental results.

Furthermore, Fig. 6 clarifies that the theoretical maximum temperature change of the magnetic working substance is approximately $3.2^{\circ} \mathrm{C}$. In comparison with this value, the experimental results shown in Fig.5 show that temperature change attains only a level of about $40 \%$ to a theoretical value. This main reason seems to be that because the gadolinium used in the experiment contains small amounts of iron and aluminum as impurities, the magnetocaloric effect at Curie point was less than that of pure gadolinium shown by theoretical calculations. In addition, it is considered that the influence of eddy current loss cannot be disregarded as well as heat loss in this present condition.

\subsection{Temperature distribution of air at cyclic process}

Figure 7 shows the respective analytical results for both the air and the magnetic working substance temperature $T_{f a n}$ and $T_{s}$ an at distance $x=0 \mathrm{~m}$ from the inlets of flow paths under the condition of the airflow rate $F=3.3 \times 10^{-5} \mathrm{~m}^{3} \mathrm{~s}^{-1}$, the initial temperature of air $T_{0}=20^{\circ} \mathrm{C}$, the heat exchange time $t_{e}=30 \mathrm{~s}$, the packing length of the working substance $L_{m}=60 \times 10^{-3} \mathrm{~m}$, and the packing rate of the working substance $\eta$ $=0.30$. The horizontal axis of the figure indicates the elapsed time from the starting time of the first cycle to the ending time of the sixth cycle. This figure reveals that the temperature change appears immediately after the demagnetization process and the magnetization process. Subsequently, both the air and the magnetic working substance temperature tend to quickly approach the initial temperature $T_{0}$, because the air temperature is near the initial temperature around the inlets of the flow paths. In addition, the temperature difference between the air and the working substance is large. Because of the heat transfer rate between the air and the magnetic working substance per unit time becomes larger.

On the other hand, Fig. 8 shows the distribution of the air temperature and the magnetic working substance temperature at the position of $x=60 \times 10^{-3} \mathrm{~m}$ over elapsed time. The heat transfer rate and the temperature difference between the air and the magnetic working substance near the outlet is smaller than the inlet section. Hence, both the air and the magnetic working substance temperature after each processes proceed to the next processes without returning to the initial temperature as shown in this figure.

\subsection{Effect of packing length on cooling rate}

The relationship between cooling rate, cooling energy and packing length of the working substance is shown in Fig. 9 for $F=8.3 \times 10^{-5} \mathrm{~m}^{3} \mathrm{~s}^{-1}, T_{0}=20^{\circ} \mathrm{C}, t_{e}=30 \mathrm{~s}$, and $\eta=0.30$. Cooling rate $q_{e x}$, as obtained through experimentation, is plotted for reference. As shown in this figure, it is found that the cooling rate and the cooling energy increase with increasing the packing length of the working substance. This tendency is quite simple; an increase in the packing length brings about an increase in the mass of the magnetic working substance directly. As a result, the cooling rate and the cooling energy defined by Eq.(2) are proportional to the packing length of the working substance. Furthermore, the figure shows that the cooling rate is maximal for working-substance packing length of ca. $L_{m}=90 \times 10^{-3} \mathrm{~m}$ as well as cooling energy. In 
these analytical conditions, because the air temperature and the working substance temperature attain an equilibrium state during the demagnetization process, heat absorption is completed at $L_{m}=90 \times 10^{-3} \mathrm{~m}$.

\subsection{Effect of particle diameter}

Figure 10 shows the temperature distribution of air over elapsed time in $T_{0}$ $=20^{\circ} \mathrm{C}, t_{e}=30 \mathrm{~s}, F=8.3 \times 10^{-5} \mathrm{~m}^{3} \mathrm{~s}^{-1}, \eta=0.10$, and $L_{m}=60 \times 10^{-3} \mathrm{~m}$. In this figure, the respective particle diameters $d_{p}$ of the working substance are $1.0 \times 10^{-3} \mathrm{~m}, 2.0 \times 10^{-3} \mathrm{~m}$, and $3.0 \times 10^{-3} \mathrm{~m}$. The major tendency through the Fig. 10 appears to be that the larger the particle diameter of the working substance, the smaller the change of air temperature after demagnetization and magnetization. When the working substance particle is larger, the gap velocity between particles decreases and the heat transfer between air and the working substance is suppressed. Consequently, the heat transfer area between the working substance and air per unit volume decreases. Furthermore, the difference between the starting temperature of the magnetization process and the initial temperature increases with an increase in the working substance particle diameter. When the working substance particles are larger, as previously described, heat transfer between air and the working substance is suppressed. For that reason, the required time for completion of heat exchange is longer than that by small particles.

Figure 11 depicts the cooling rate $q_{a n}$ as a function of the packing rate $\eta$. In this figure, the working substance particle diameters $d_{p}$ are $1.0 \times 10^{-3} \mathrm{~m}, 2.0 \times 10^{-3} \mathrm{~m}$ and $3.0 \times 10^{-3} \mathrm{~m}$, respectively. The general feature of this figure shows the fact that the cooling rate with respect to the working substance particle diameter increases when the working substance packing rate increases. This may be the reason why the fact that the cooling energy of the working substance in the flow paths increases because the working substance mass is higher for an increased packing rate of the working substance. Beside, the gap velocity of the heat transfer medium also increases with the working substance packing rate. Thereby, the heat transfer coefficient increases.

For working substance packing rates of less than about 0.20 , the larger the working substance particles, the greater the cooling rate. This is also explained by considering the fact that the cooling rate tends to be higher than that of smaller working substance particles. Namely, the starting temperature of demagnetization becomes lower than the initial temperature for larger particles. On the other hand, in a condition in which the working substance packing is greater than about 0.20 , larger particles have a lower cooling rate. This is caused by the fact that the heat transfer improves markedly according to the reduced particle diameter when the packing rate of the working substance is high.

\section{Conclusions}

Regarding the room-temperature magnetic refrigeration system, cooling characteristics and optimum operating conditions have been determined both experimentally and analytically. The following conclusions may be drawn within the parameter range covered in the present study.

(1) Cooling rate is highest when setting the Curie point of the magnetic working substance to the starting temperature.

(2) The analytical model constructed in this study realizes the experimental results and 
confirms the propriety of this model.

(3) When the working substance particles are large, the temperature change in one cycle is small.

(4) Regarding the packing rate and packing length of the working substance: if an appropriate packing rate and the packing length are selected, it is possible to obtain cooling rate beyond typical maximum values.

\section{Acknowledgements}

This work has been supported financially by the Japan Society for the Promotion of Science under the Grant-in-Aid for Scientific Research (Young Scientists 16760146). We thank Y. Isono for his active experimental work.

\section{References}

[1] Brown, G.V., 1976, Magnetic Heat Pumping near Room Temperature, J. Appl. Phys., vol.47, no.8 : p.2110-2112.

[2] Benford, S.M., Brown, G.V., 1981, T-S Diagram for Gadolinium near the Curie Temperature, J. Appl. Phys., vol.52, no.3 : p.3673-3680.

[3] Pecharsky, V.K., Gschneidner, Jr., K.A., 1999, Magnetocaloric Effect and Magnetic Refrigeration, J. Magnetism and Magnetic Materials, vol.200 : p.44-56.

[4] Gschneidner, Jr., K.A., Pecharsky, V.K., 1999, Magnetic Refrigeration Materials, J. Appl. Phys., vo.85, no.8 : p.5365-5368.

[5] Xu, Y., Meier, M., Das, P., Koblischka, M.R., Hartmann, 2002, U., Perovskite Manganites : Potensial Materials for Magnetic cooling at or near Room Temperature, Crystal Engineering, vol.5 : p.383-389.

[6] Brück, E., 2003, Magnetic Refrigeration - towards Room-temperature applications, Physica, vol.B, no.327 : p.431-437.

[7] Hashimoto, T., Numasawa, T., Shino, M., Okada, T., 1981, Magnetic Refrigeration in the Temperature Range from $10 \mathrm{~K}$ to Room Temperature : the Ferromagnetic Refrigerants, Cryogenics, Nov. : p.647-653.

[8] Ranz, W.E, 1952, Friction and Transfer Coefficient for Single Particles and Packed Beds, Chemical Engineering Progress, vol.48, no.5 : p.247-253. 


\section{Figure captions}

Figure 1: Schematic of experimental apparatus

Figure 2: Details of test section

Figure 3: Simulation model ; (a) Coordinate system, (b) Cross section in X-Y plane

Figure 4: Temperature change of magnetic working substance

Figure 5: Experimental results on air temperature

Figure 6: Numerical results on air temperature

Figure 7: Temperature distribution of air and magnetic working substance $(x=0 \mathrm{~m})$

Figure 8: Temperature distribution of air and magnetic working substance ( $x$ $\left.=60 \times 10^{-3} \mathrm{~m}\right)$

Figure 9: Effect of packing length

Figure 10: Numerical results on air temperature

Figure 11: Effect of packing rate on cooling rate 


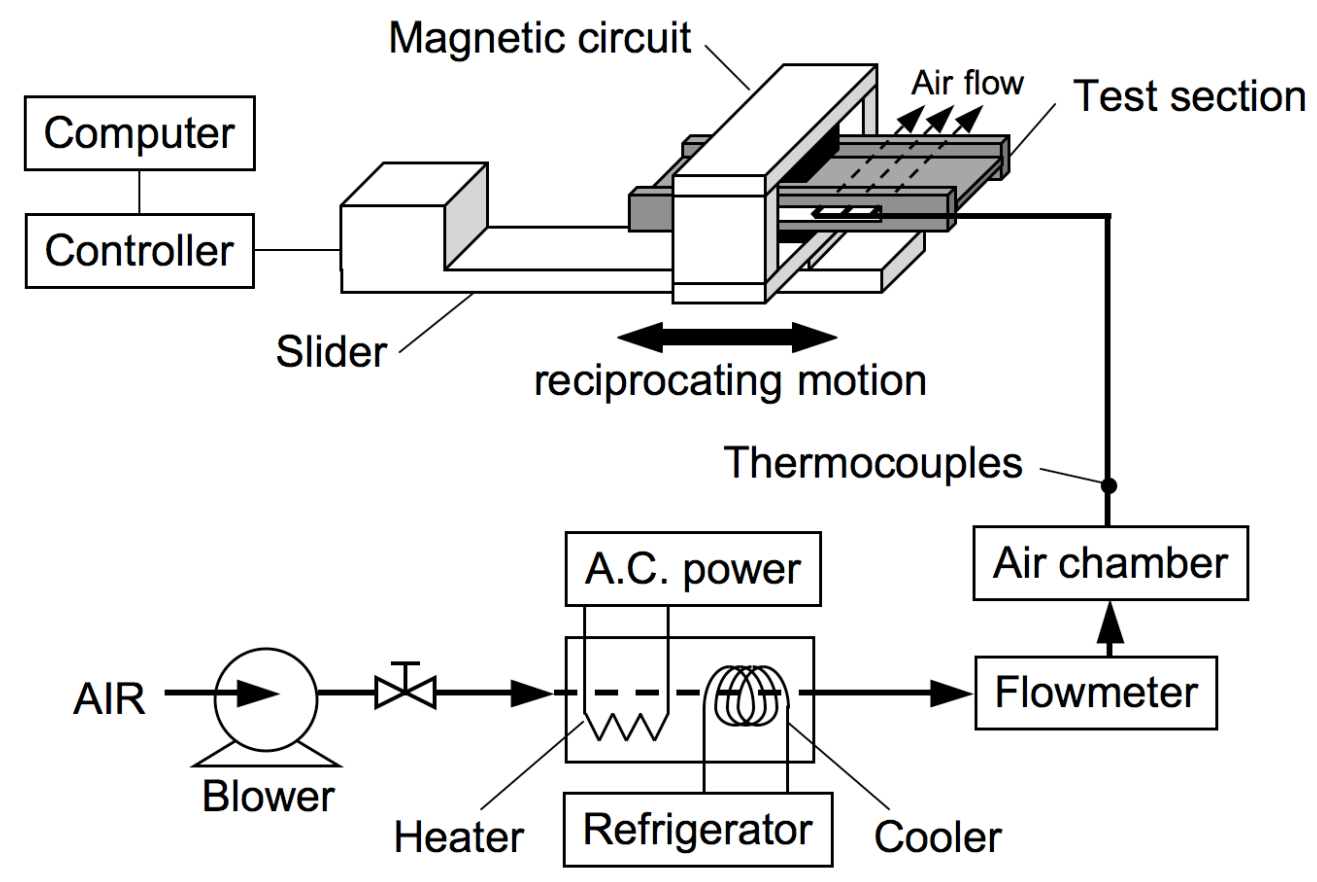

Figure 1 


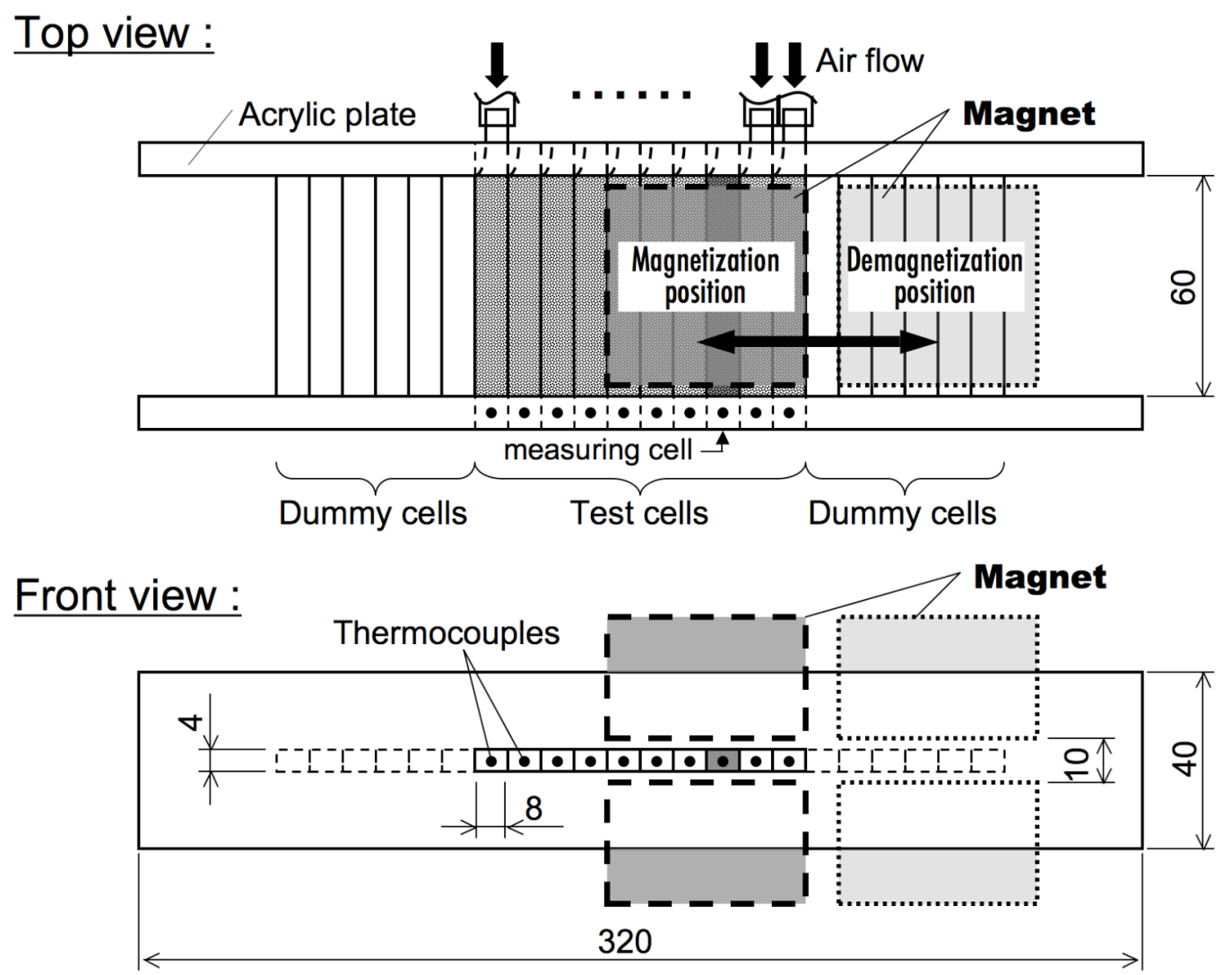

Figure 2 


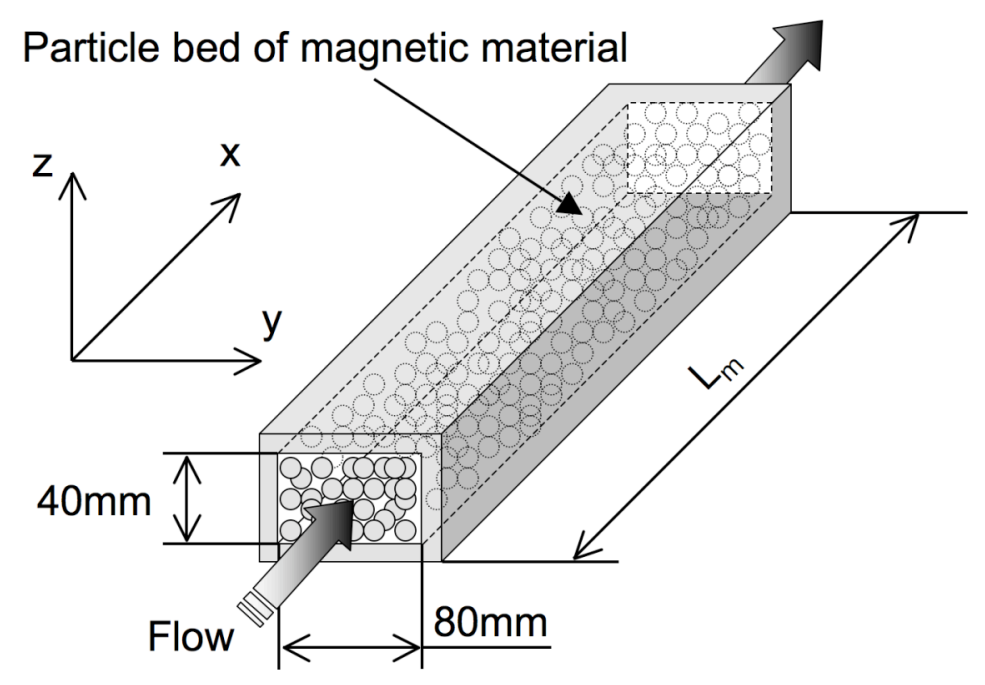

(b)

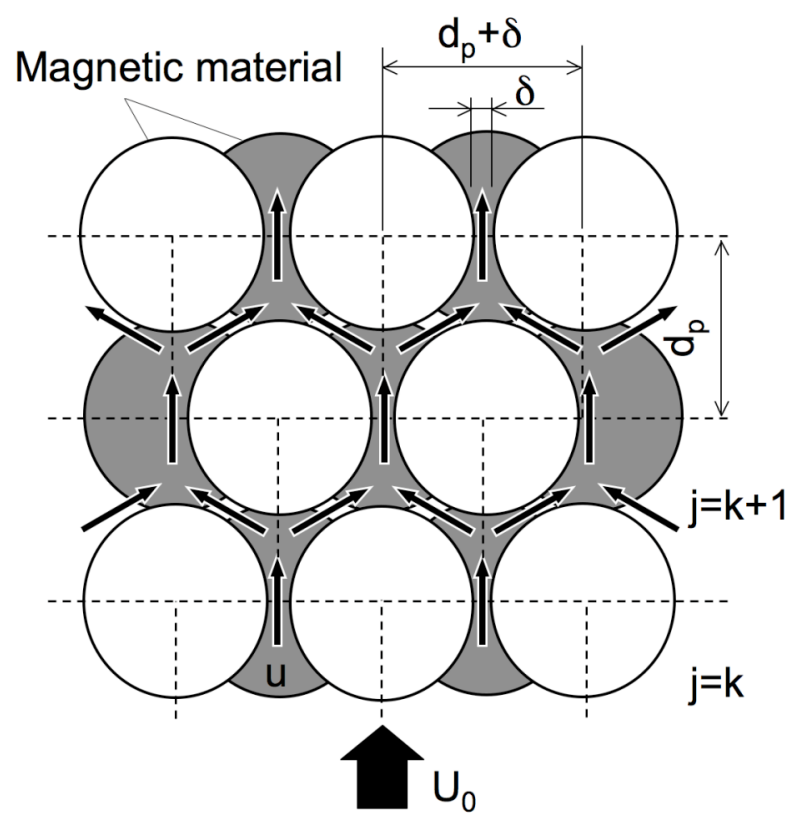




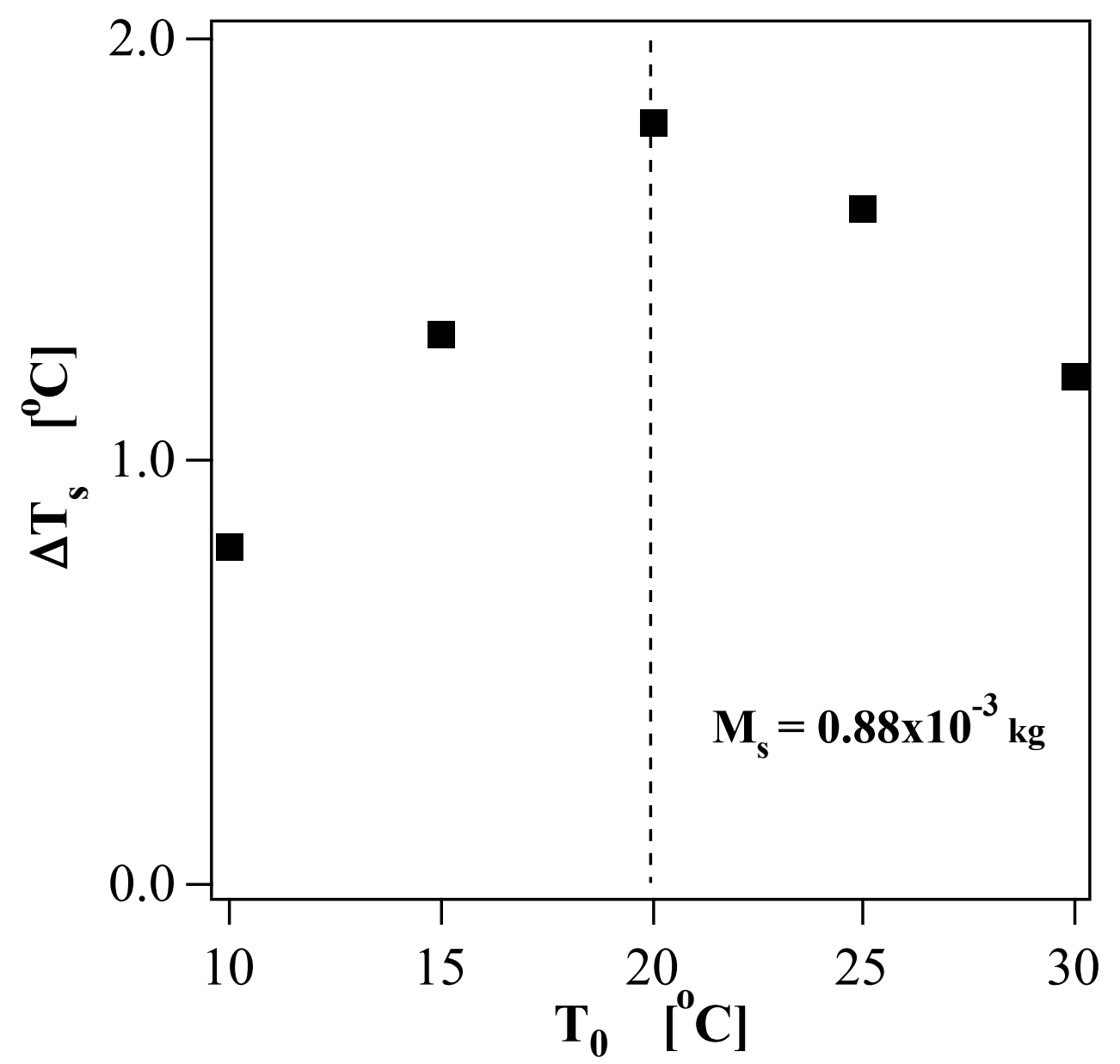

Figure 4 


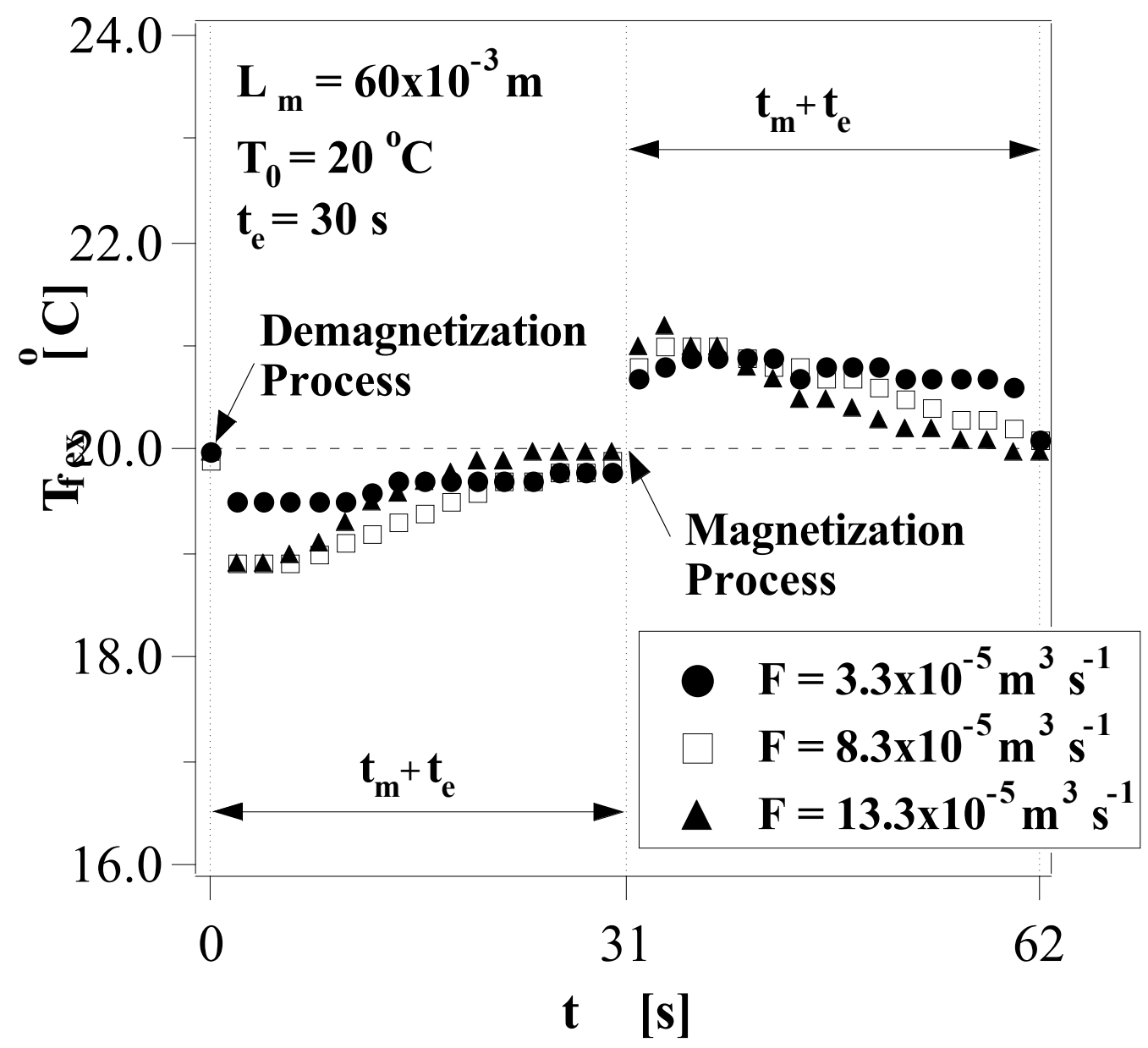

Figure 5 


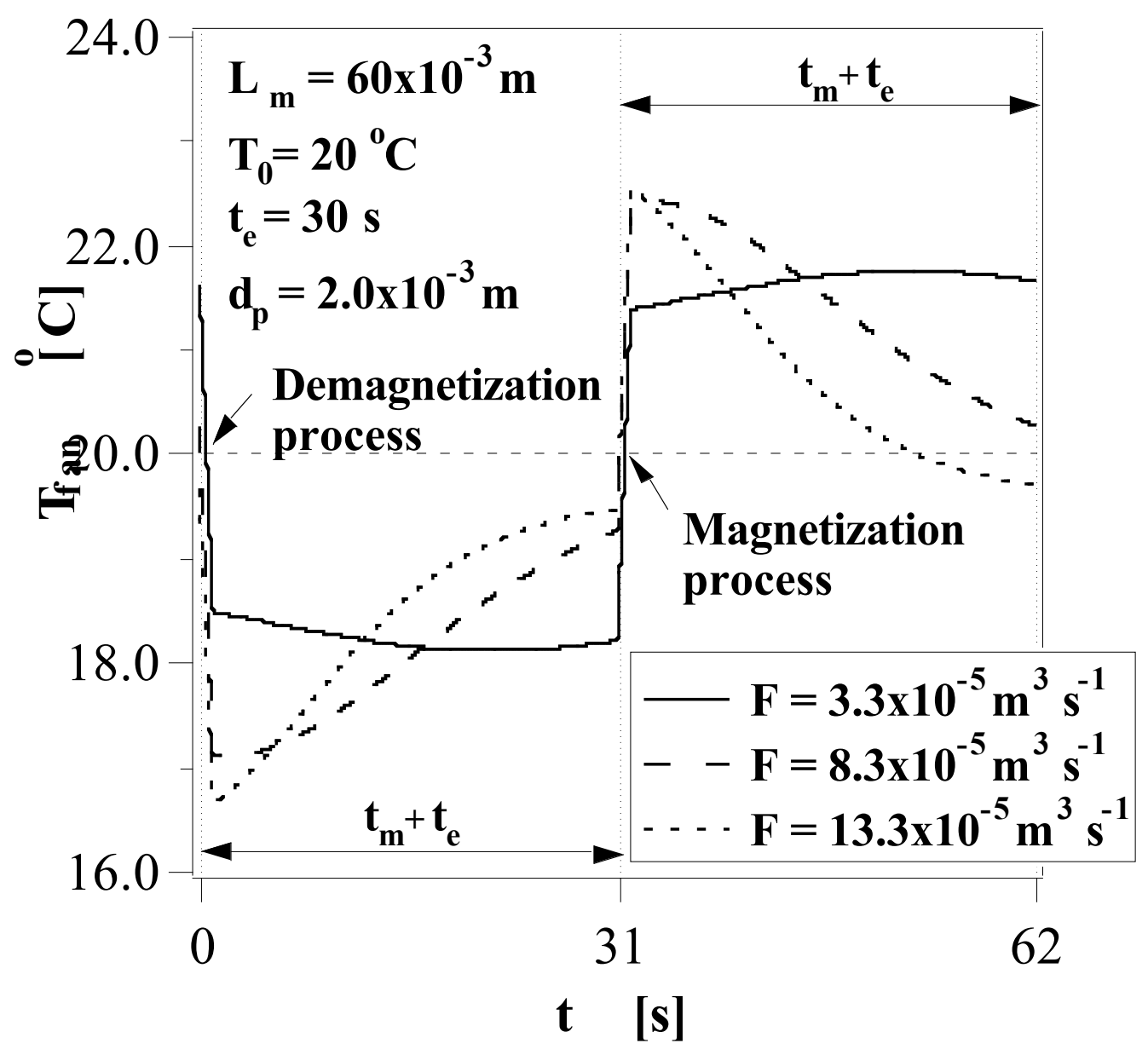

Figure 6 


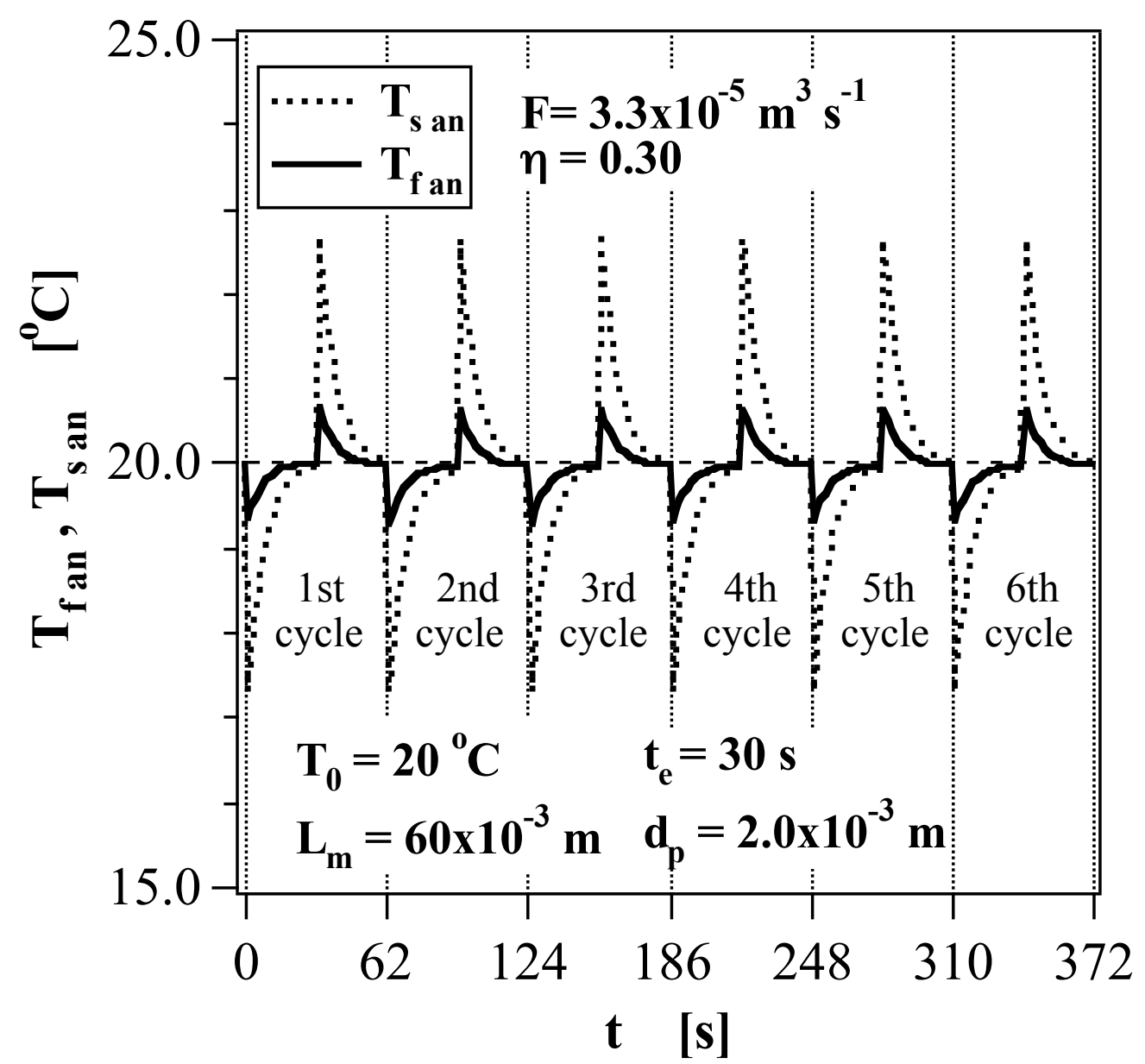

Figure 7 


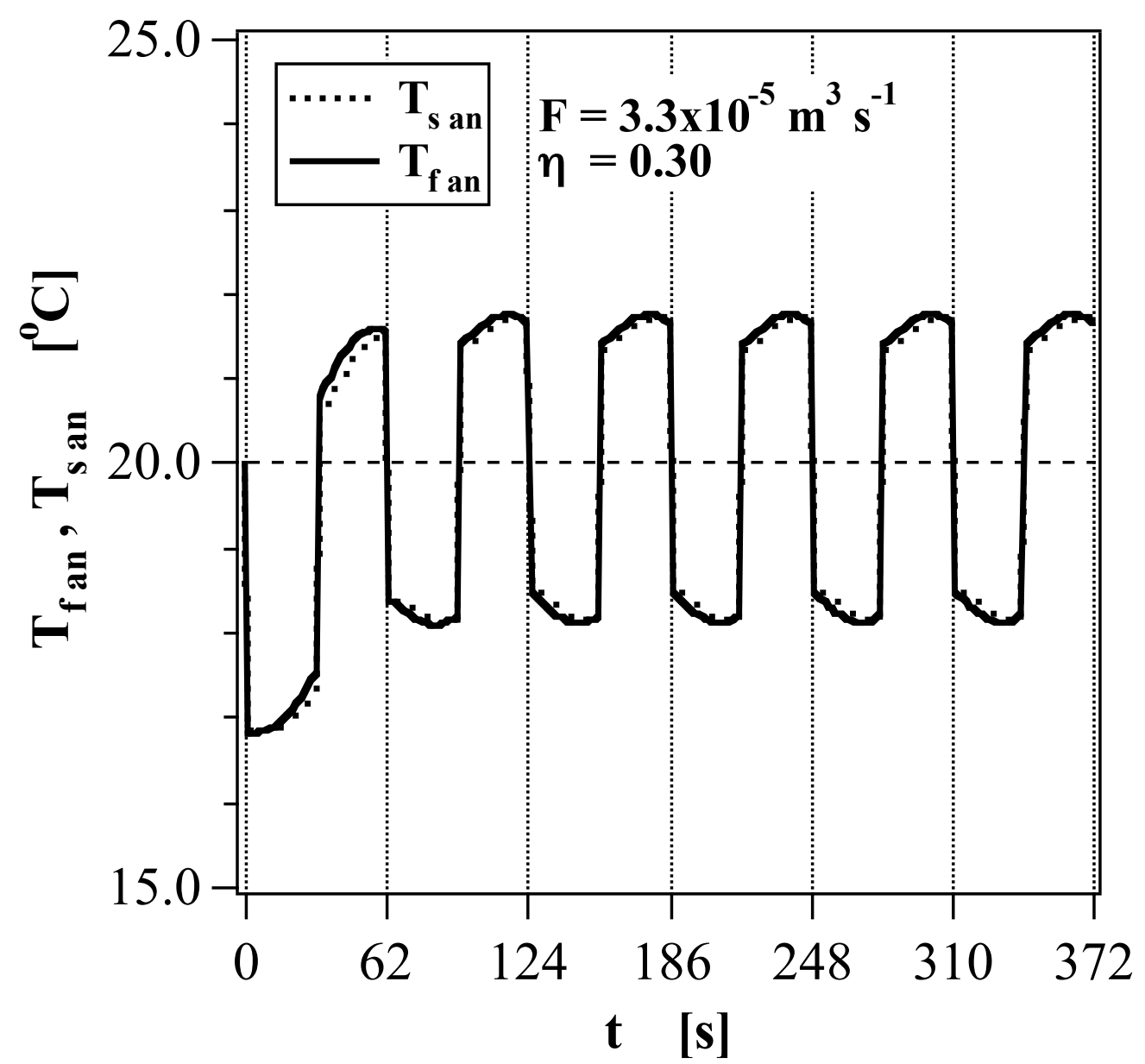

Figure 8 


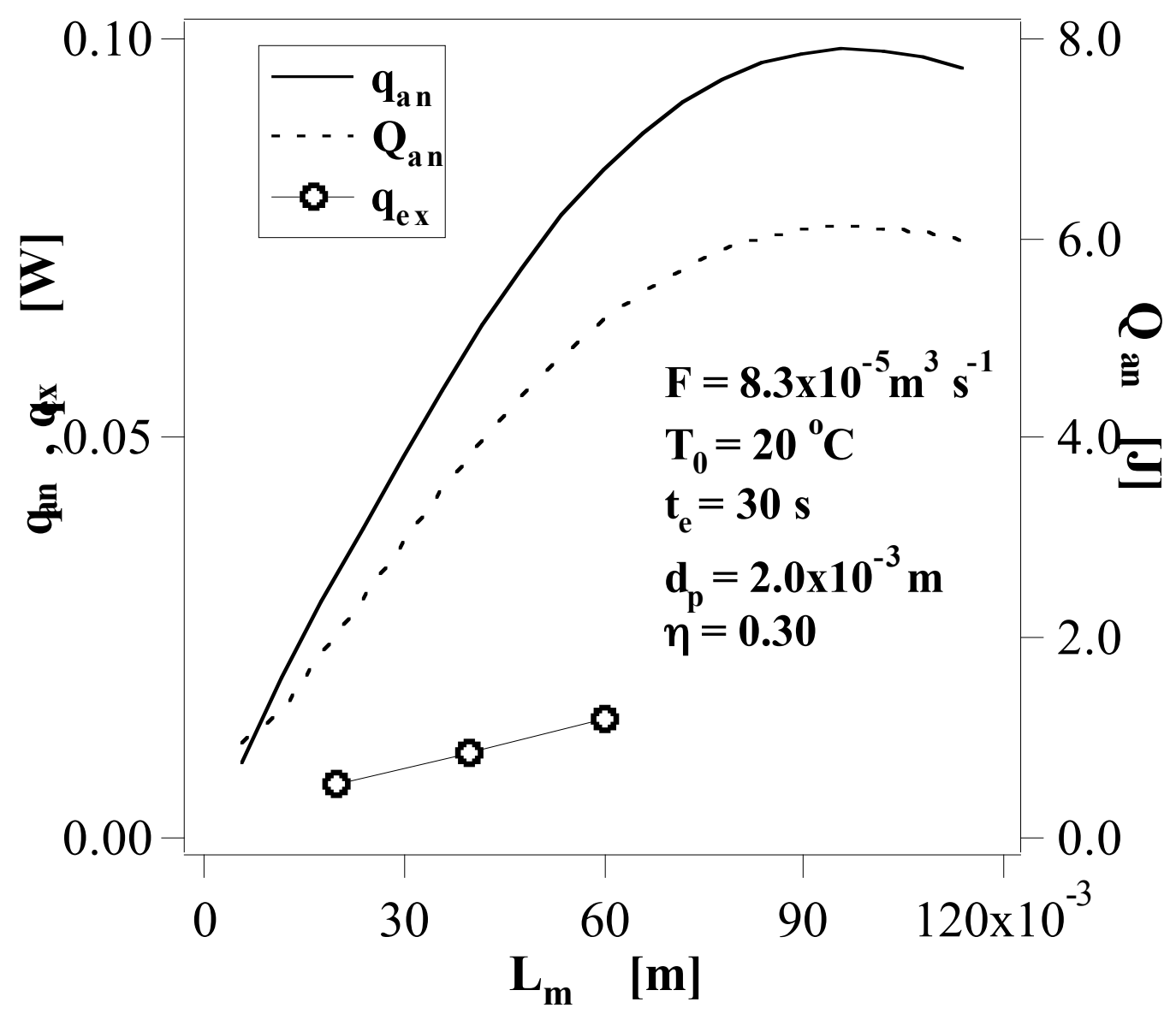

Figure 9 


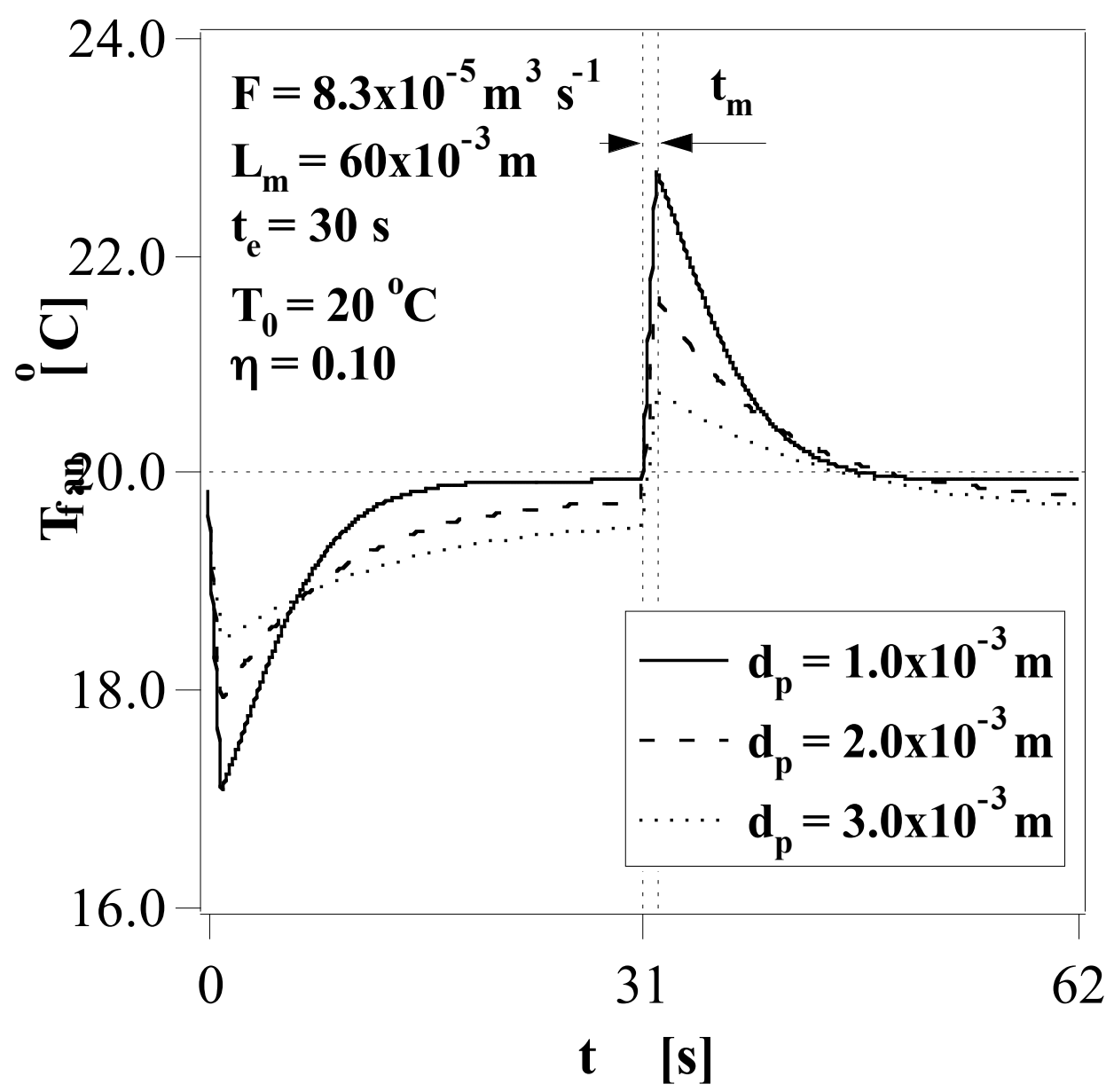

Figure 10 


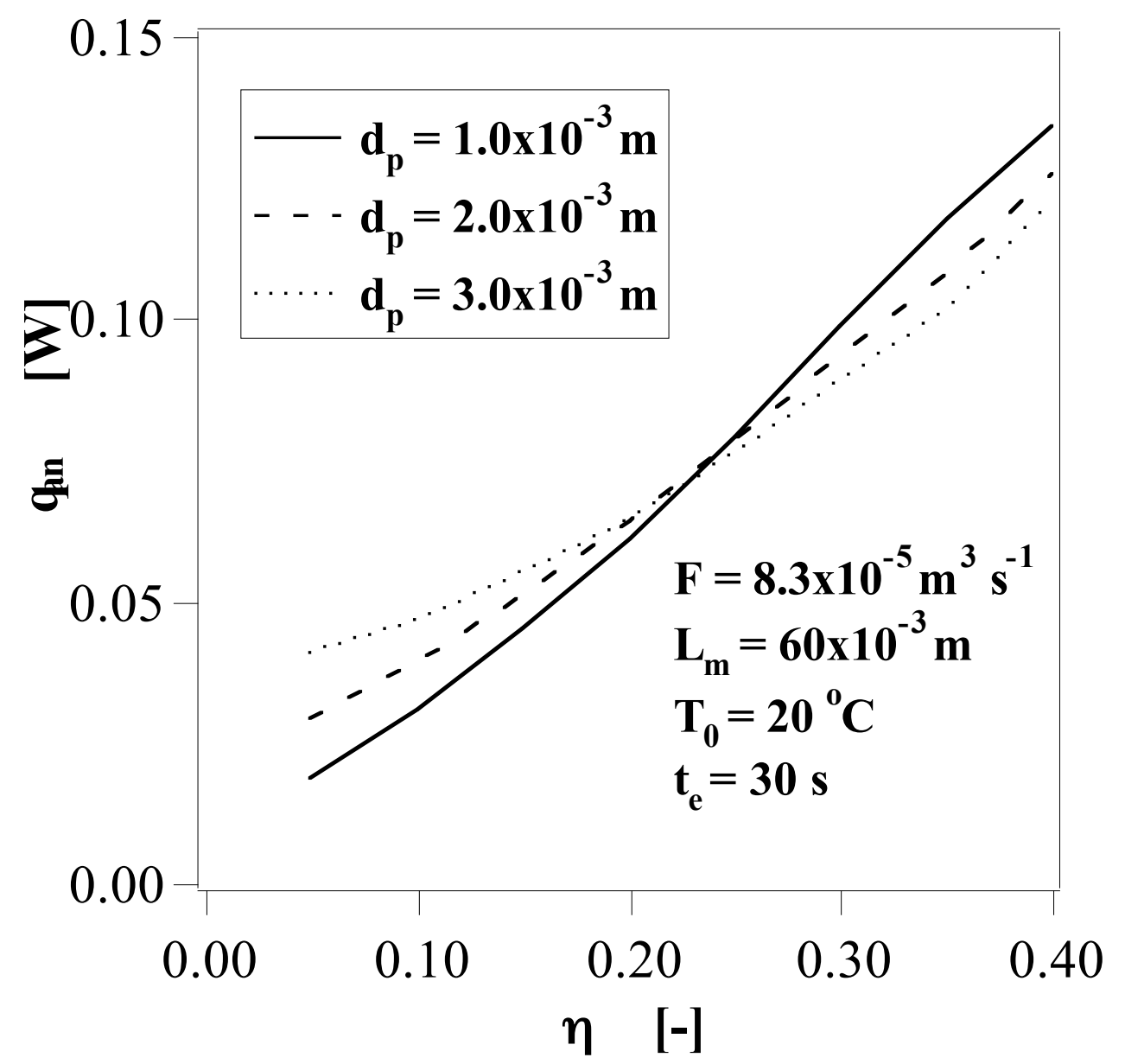

Figure 11 